\title{
Monitoring of historical frescoes by timed infrared imaging analysis
}

\author{
G. CADELANO ${ }^{* 1}$, P. BISON ${ }^{1}$, A. BORTOLIN ${ }^{1}$, G. FERRARINI ${ }^{1}$, F. PERON ${ }^{2}$, M. GIROTTO ${ }^{3}$, \\ and M. VOLINIA ${ }^{3}$
}

\author{
${ }^{1}$ Consiglio Nazionale delle Ricerche - ITC, Corso Stati Uniti 4, 35127 Padova, Italy \\ ${ }^{2}$ Universita Iuav di Venezia - Santa Croce 191 Tolentini, 30135 Venezia, Italy \\ ${ }^{3}$ Politecnico di Torino, Corso Duca degli Abruzzi 24, 10129 Torino, Italy
}

\begin{abstract}
The subflorescence and efflorescence phenomena are widely acknowledged as the major causes of permanent damage to fresco wall paintings. They are related to the occurrence of cycles of dry/wet conditions inside the walls. Therefore, it is essential to identify the presence of water on the decorated surfaces and inside the walls.

Nondestructive testing in industrial applications have confirmed that active infrared thermography with continuous timed images acquisition can improve the outcomes of thermal analysis aimed to moisture identification. In spite of that, in cultural heritage investigations these techniques have not been yet used extensively on a regular basis. This paper illustrates an application of these principles in order to evaluate the decay of fresco mural paintings in a medieval chapel located in North-West of Italy. One important feature of this study is the use of a robotic system called aIRview that can be utilized to automatically acquire and process thermal images. Multiple accurate thermal views of the inside walls of the building have been produced in a survey that lasted several days. Signal processing algorithms based on Fast Fourier Transform analysis have been applied to the acquired data in order to formulate trustworthy hypotheses about the deterioration mechanisms.
\end{abstract}

Keywords: infrared thermography, thermal imaging, fresco detachment, moisture detection, cultural heritage monitoring, building survey.

\section{Introduction}

The preservation of cultural heritage requires the knowledge of phenomena involved in the process of decay of artworks. The basic materials for a fresco are products derived from limestone. Fresco is made of calcium carbonate that forms a very hard and stable surface trapping the pigments. Even if fresco is one of the oldest and most durable painting techniques, it is subject to deterioration due to several agents, such as biological or mechanical damages caused by water. Efflorescence and subflorescence [1] are generally appointed as the most dangerous causes of decay. In the field of building conservation, the term efflorescence describes those mineral deposits that occur on the surface of stone masonry, bricks, plasters or other architectural elements. These crystals are generated as a result of evaporation of water in which salts were dissolved. The crystals can be formed both inside and outside of a surface, the latter are often related to conditions of fast water evaporation and are defined as subflorescence. Subflorescence is considered a much more dangerous phenomenon in respect to superficial efflorescence because the volume change of the salts can lead to mechanical damages inside the wall, causing the detachment of parts of the intonaco layer. Consequently, it is paramount to detect the presence of water on the deco-

\footnotetext{
*e-mail: gianluca.cadelano@itc.cnr.it
}

rated surfaces and inside the walls before the decay mechanism starts. Moreover, it is fundamental to discover the source and the behaviour of the water over time, especially in relation to dynamic environmental conditions that may occur during the year. These can be sometimes predictable, as the effect of visiting people on the air moisture content, or unpredictable as seasonal weather changes [2,3]. Even though quantitative local methods, such as thermogravimetric analysis of wall samples [4], have proven to get precise information concerning the moisture content of the building materials, in most circumstances these methodologies are not appropriate, and often their application to artworks is not allowed due to their invasive or destructive characteristics.

An alternative method to investigate the presence of water inside porous materials and artworks is observing its thermal effect by measuring the surface temperature with appropriated techniques such as infrared thermography (IRT) [5].

IRT is a contactless and nondestructive technique which, with the aid of a thermal camera, allows to measure and display temperature of the surface of an object by using the measurement of the infrared radiation emitted by the surface itself $[6,7]$.

The typical raw data output of IRT is a temperature matrix, the size of which is correlated with the technological characteristics of the sensor. 
IRT is widely used in many fields where thermal signals are of interest, such as condition monitoring for civil and industrial components, defect detection, and material characterization [8].

IRT is widespread in the building inspection field for the evaluation of temperature distribution and heat losses of the building envelope [9]. Literature shows that infrared thermography is also a valuable instrument for accurate thermal analysis of historical architectures in order to monitor indoor and outdoor surfaces and to evaluate the distribution of moisture $[10,11]$. Furthermore, this technique is promising for the study of works of art such as fresco paintings [12-14].

The most basic building survey by infrared thermography is often restricted to the acquisition of single shot images taken in a limited range of time. Extending the examination in space and time makes it possible to get much more information. This can be achieved observing wider areas through the mosaicking technique commonly applied in photography for panorama views $[15,16]$.

Moreover, the acquisition of multiple time lapsed images can easily increase the total observation time, allowing to follow dynamically varying phenomena. These methods require a technical effort, since ordinary commercially available thermographic systems cannot perform these operations as the needed functions are not provided. A robotized system called aIRview, developed at CNR-ITC in Padua, Italy, is a solution to study buildings in transient conditions. At this time aIRview has been set to operate in indoor environment. In this application the acquired thermal data are mostly related to day/night cycle, and can be processed as in the lock-in thermography method, that is now considered a suitable technique for the detection and quantification of defects in industrial applications [17]. The aim of this study is to demonstrate the usefulness of an advanced infrared based acquisition system combined with active timed thermography technique, in pushing forward the knowledge of moisture related decay affecting the historical murals.

\section{Case study}

St. Eldrad's chapel dates back to the $10^{\text {th }}$ or $11^{\text {th }}$ century, and it is a part of the Abbey of Novalesa complex located about $60 \mathrm{~km}$ West of Turin, on the Italian border with France. The structure is not easily accessible and measures $8 \times 5.8 \times 3$ meters inside. The interior walls and ceiling are entirely adorned with mural paintings that go back to the second half of the $11^{\text {th }}$ century. The decorations were executed by means of the fresco technique, and portray religious themes and the story of St. Nicholas and St. Eldrad. It has been testified by art historians that these beautiful frescoes did not suffered any particular problem of conservation since their painting until modern times. Indeed, after recent works, severe damage began to appear, mainly on the lower side of the southern wall. Documents regarding latest years are not exhaustive and sketchy, but it is possible to deduce that some restoration occurred. The plaster layer of the outer wall has been removed from all the surfaces. Moreover, a window on the South side that was closed in ancient times was opened. The hallmark shape of the boundaries of the ruined areas and the extensive presence of efflorescence could be related to wall moisture phenomena, but the source of the water and the reason why the wall facing south is by and large the most damaged are unknown.

\section{Experimental procedure}

The experiment is an active thermography procedure applied to the South wall of the chapel. Here the thermal source is the solar radiation that can be considered as a periodic stimulus $\cos (\omega t)$, where $\omega=2 \pi / \tau$ is the angular frequency, $t$ is the time, and $\tau$ is the period equal to 24 hours. As a consequence, a thermal wave propagates from the outer surface of the building to the inside of the chapel. Observing the wall from inside, assuming evenly space of distributed solar radiation, constant thickness, and homogeneous properties of the wall, water content is expected to have a key role in the observed thermal anomalies. Considering the thermal conductivity, the mass density, and the specific heat of the dry material (respectively $\lambda_{d r y}, \rho_{d r y}$, and $c_{d r y}$ ) as reference values for wall materials taken from literature, the thermophysical properties (respectively, $\lambda_{\text {eff }}, \rho_{\text {eff }}$, and $c_{\text {eff }}$ ) of the same wall in wet condition can be approximated [18,19] taking into account the effect of water as a function of the ratio between the volume of moisture in the porosity otherwise filled by air and the total volume of the wall, as shown in Eqs. (1) and (2)

$$
\begin{gathered}
\frac{\lambda_{d r y} \lambda_{\text {water }}}{\lambda_{\text {water }}+\lambda_{\text {dry }} \Phi_{\frac{\text { water }}{\text { total }}}} \leq \lambda_{\text {eff }} \leq \lambda_{\text {dry }}+\lambda_{\text {water }} \Phi_{\frac{\text { water }}{\text { total }}}, \\
\rho_{\text {eff }} c_{\text {eff }}=\frac{P_{\text {total }}}{V_{\text {total }}}\left[c_{d r y} \frac{P_{d r y}}{P_{\text {tot }}}+c_{\text {water }} \frac{P_{\text {water }}}{P_{\text {total }}}\right]= \\
=c_{d r y} \rho_{\text {dry }}+c_{\text {water }} \frac{P_{\text {water }}}{V_{\text {water }}} \frac{V_{\text {water }}}{V_{\text {total }}}= \\
=c_{d r y} \rho_{d r y}+c_{\text {water }} \rho_{\text {water }} \Phi_{\frac{\text { water }}{\text { total }}},
\end{gathered}
$$

where:

$\Phi_{\underline{w a t e r}}$ is the ratio between the volume of moisture in the porosity otherwise filled by air and the total volume of the wall;

$\lambda_{d r y}$ is the thermal conductivity of dry wall materials; $\lambda_{\text {water }}$ is the thermal conductivity of water;

$\rho_{d r y}$ is the mass density of dry wall materials;

$\rho_{\text {water }}$ is the mass density of water;

$P_{d r y}$ is the mass of dry wall materials;

$P_{\text {water }}$ is the mass of water in the wall;

$P_{\text {total }}$ is the mass of the wall;

$c_{d r y}$ is the specific heat of dry wall materials;

$c_{\text {water }}$ is the specific heat of water;

$V_{\text {water }}$ is the volume of water in the wall;

$V_{\text {total }}$ is the volume of the wall. 


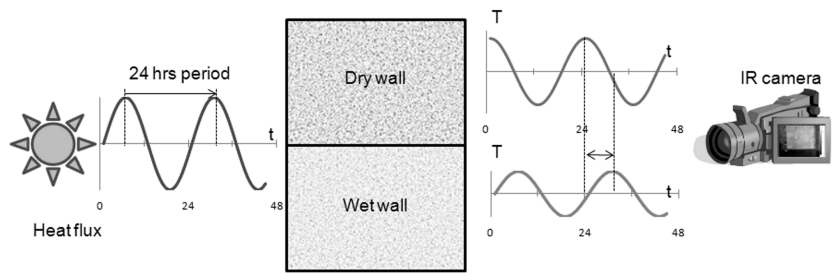

Fig. 1. Experimental setup schematization. In order to collect information about the effect of dry and wet materials on the thermal waves behaviour, temperature variation of the inside surface is recorded over time.

The inner surface temperature oscillates with the same period of the solar heat flux. The amplitude of the wave depends on the thermophysical properties of the wall; its phase, as well as schematized in Fig. 1. After recording the inner surface temperature over several days, a Fast Fourier Transform of the temperature signal has been computed for every pixel [20], retrieving amplitude and phase at the frequency corresponding to a period equal to 24 hours [21].

By Eqs. (1) and (2) it is possible to calculate the thermal diffusivity values for both dry [Eq. (3)] and wet [Eq. (4)] conditions, and the expected penetration depth of thermal waves at a given time.

$$
\begin{aligned}
& \alpha_{d r y}=\frac{\lambda_{d r y}}{(\rho c)_{d r y}} ; \mu_{d r y}=\sqrt{\frac{2 \alpha_{d r y}}{\omega}}, \\
& \alpha_{w e t}=\frac{\lambda_{w e t}}{(\rho c)_{w e t}} ; \mu_{w e t}=\sqrt{\frac{2 \alpha_{w e t}}{\omega}} .
\end{aligned}
$$

Where:

$\alpha_{d r y}$ is the thermal diffusivity of the wall material in dry condition;

$\mu_{d r y}$ is the thermal wave penetration depth of the wall material in dry condition;

$\alpha_{w e t}$ is the thermal diffusivity of the wall material in wet condition; $\mu_{\text {wet }}$ is the thermal wave penetration depth of the wall material in wet condition.

The following Eq. (5) represents the behaviour of the thermal wave as a function of depth $x$ and time $t$

$$
T(x, t) \propto A e^{-x / \mu} \cos (\omega t-x / \mu)
$$

$\mu$ assumes the value $\mu_{d r y}$ or $\mu_{w e t}$ depending on moisture conditions. The amplitude of the thermal wave is exponentially decreased and its phase is linearly delayed, depending on $\mu$, that on its turn depends on the water content as in Eqs. (3) and (4). Comparing the reference values, shown in Table 1 , it is evident that despite variations on thermal conductivity and mass density, the specific heat plays a key role in the thermal wave propagation mechanism governed by thermal diffusivity $(\alpha)$. Since the heat capacity $(\rho c)$ of water is sensibly greater than the heat capacity of the considered construction materials, the resulting hypothesis is that thermal waves that pass through the wet material should be attenuated (have a lower amplitude) and phase shifted compared to the thermal wave passing though the dry material.

\section{Mathematical simulations}

The wall structure has been simply modelled in small scale to proportionally replicate the real phenomena, in bricks, assuming the thermophysical properties of this material from the values of Table 1 and a thickness equal to $0.05 \mathrm{~m}$.

In order to model four different moisture contents at constant thickness, part of the voids volume has been progressively replaced with water, moving from dry condition to saturation (40\% water content) as in Table 1

Clay bricks - dry (represents the ideal dry wall),

Clay bricks - $10 \%$ water content;

Clay bricks - $20 \%$ water content;

and Clay bricks $-40 \%$ water content (represents the saturated wall).

The solar thermal stimulus has been scaled too and has

\begin{tabular}{|c|c|c|c|c|c|}
\hline Material & $\lambda\left[\mathrm{W} \mathrm{m}^{-1} \mathrm{~K}^{-1}\right]$ & $\rho\left[\mathrm{kg} \mathrm{m}^{-3}\right]$ & $c\left[\mathrm{~J} \mathrm{~kg}^{-1} \mathrm{~K}^{-1}\right]$ & $C\left[\mathrm{~kJ} \mathrm{~K}^{-1} \mathrm{~m}^{-3}\right]$ & $\alpha\left[\mathrm{m}^{2} \mathrm{~s}^{-1}\right]$ \\
\hline Clay bricks - dry ${ }^{1}$ & 0.5 & 1800 & 840 & 1512 & $3.31 \mathrm{E}-07$ \\
\hline Clay bricks $-10 \%$ water content ${ }^{2}$ & 0.56 & 1900 & 1016 & 1903 & $2.90 \mathrm{E}-07$ \\
\hline Clay bricks $-20 \%$ water content ${ }^{2}$ & 0.62 & 2000 & 1174 & 2348 & 2.64E-07 \\
\hline Clay bricks $-40 \%$ water content ${ }^{2}$ & 0.74 & 2200 & 1448 & 3185 & 2.32E-07 \\
\hline Marble - dry ${ }^{1}$ & 2.8 & 2600 & 800 & 2080 & $1.35 \mathrm{E}-06$ \\
\hline Marble $-5 \%$ water content ${ }^{2}$ & 2.85 & 2650 & 864 & 2290 & $1.24 \mathrm{E}-06$ \\
\hline Pietra Serena (sandstone) - dry 1 & 2.45 & 2540 & 811 & 2060 & $1.19 \mathrm{E}-06$ \\
\hline Pietra Serena (sandstone) $-5 \%$ water content ${ }^{2}$ & 2.5 & 2590 & 876 & 2269 & $1.10 \mathrm{E}-06$ \\
\hline Water $^{3}$ & 0.6 & 1000 & 4182 & 4182 & $1.43 \mathrm{E}-07$ \\
\hline
\end{tabular}
been modelled as a periodic heat wave of period equal to

Table 1. Thermophysical properties of common construction materials.

${ }^{1}$ values measured at CNR-ITC lab

${ }^{2}$ values computed by Eqs. (1), (2), (3) and (4)

${ }^{3}$ values taken from literature [22]. 


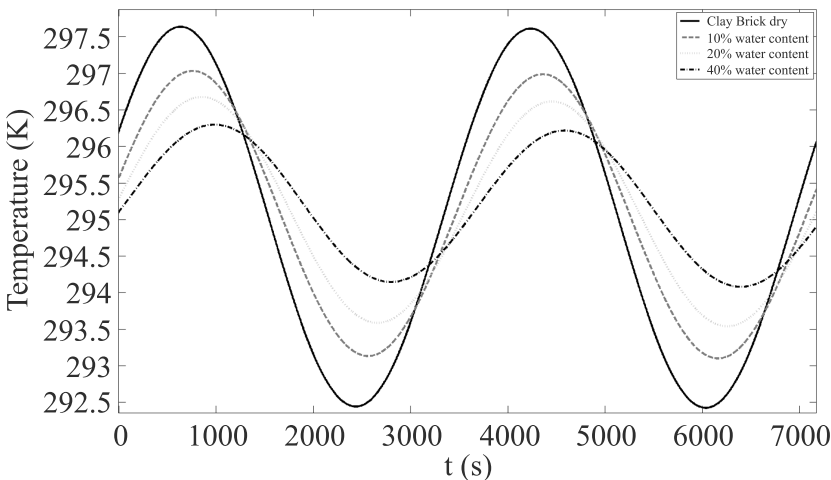

Fig. 2. Surface temperatures evolution of the modelled clay brick specimen with different water contents. As expected, both dry and wet wall surface temperatures oscillate with the same period of the imposed stimulus. The wet material waves have lower amplitude than the dry ones and are delayed in phase according to the increasing water content.

1 hour. The model has been built using Finite Element Method (FEM) [23]. The surface temperatures have been collected during the dynamic process and then analysed using FFT algorithm extracting amplitude and phase parameters. The following picture shows the thermal behaviour of the test block: the dry wall is represented in a continuous black line, three different moisture conditions are represented in dashed gray lines. Figure 2 shows the evolution of surface temperatures over two modelled periods.

The temperature profiles, as seen in Fig. 2, are usually represented in time domain. By applying Discrete Fourier Transform to the time evolution of every pixel it is possible to consider the data in frequency domain according to the following relation

$$
F_{u}=\frac{1}{N} \sum_{u=0}^{N-1} h(t) \exp ^{(-j 2 \pi u t / N)}=\operatorname{Re}\left(F_{u}\right)+j \operatorname{Im}\left(F_{u}\right)
$$

Where:

$u$ is the frequency;

$N$ is the number of images of the thermal sequence;

$j$ is the imaginary unit;

Re is the real part of the transform;

Im is the imaginary part of the transform.

Amplitude $(A)$ and Phase $(\Phi)$ are obtained as follows in Eqs. (7)

$$
A_{u}=\sqrt{\operatorname{Re}\left(F_{u}\right)^{2}+\operatorname{Im}\left(F_{u}\right)^{2}} ; \Phi_{u}=\tan ^{-1}\left[\frac{\operatorname{Im}\left(F_{u}\right)}{\operatorname{Re}\left(F_{u}\right)}\right] .
$$

The following Table 2 summarizes the results $(A$ and $\Phi)$ of the FFT algorithm applied on the modelled data.

The obtained $A$ and $\Phi$ values are represented in Table 2 for the third frequency component of the Fourier Transform which is the most important component as it is the first harmonic of the analysed signal. As expected the presence of water determines lower amplitude and a phase shift in respect to the wave describing the dry condition.
Table 2. Amplitude and Phase values at different moisture contents show a different behaviour of dry and wet simulated walls. The obtained amplitude and phase values are coherent with the theoretical expectations and so this method potentially distinguishes dry from wet conditions.

\begin{tabular}{lcc}
\hline Material & Amplitude (a.u.) & Phase (rad) \\
\hline Clay bricks & 2588 & 0.196 \\
Clay bricks - 10\% water content $^{2}$ & 1933 & 0.416 \\
Clay bricks - 20\% water content $^{2}$ & 1522 & 0.584 \\
Clay bricks - 40\% water content $^{2}$ & 1045 & 0.824 \\
\hline
\end{tabular}

\section{Data collection}

One important feature of this work is the application of an automated system called aIRview, a robotic system that can automatically acquire and process thermal images, scanning a surface at the pace of $30 \mathrm{~m}^{2}$ per minute (Fig. 3).

alRview system is a complex apparatus whose core is made of a microbolometric infrared camera working in the range from 7.5 to $13.0 \mu \mathrm{m}$ with a Noise-Equivalent Temperature Difference (NETD) of $50 \mathrm{mK}$ at $30^{\circ} \mathrm{C}$. The detector's size is $320 \times 240$ pixels. The IR camera is mounted on a pan-tilt device that allows to scan a wall by taking images of neighbouring fields of the view. The frame rate of the imaging system is limited to $0.5 \mathrm{~Hz}$ for this specific application. The frame rate accounts for the acquisition time of each IR image (in this case 20) of the whole scanning plus the time to change the field of view by panning and tilting the camera. The acquisition is automatically managed by custom Labview software while the IR image processing is done in Matlab environment. The images related to one scanning are automatically corrected for radiometric and geometric distortions and successively mosaicked to obtain a complete view of the surface of interest using a grid of special targets designed to work in the infrared range [24]. The spatial resolution of the thermal mosaic is $1 \mathrm{pixel} / \mathrm{cm}$ and its size depends on the dimension of the investigated surface.

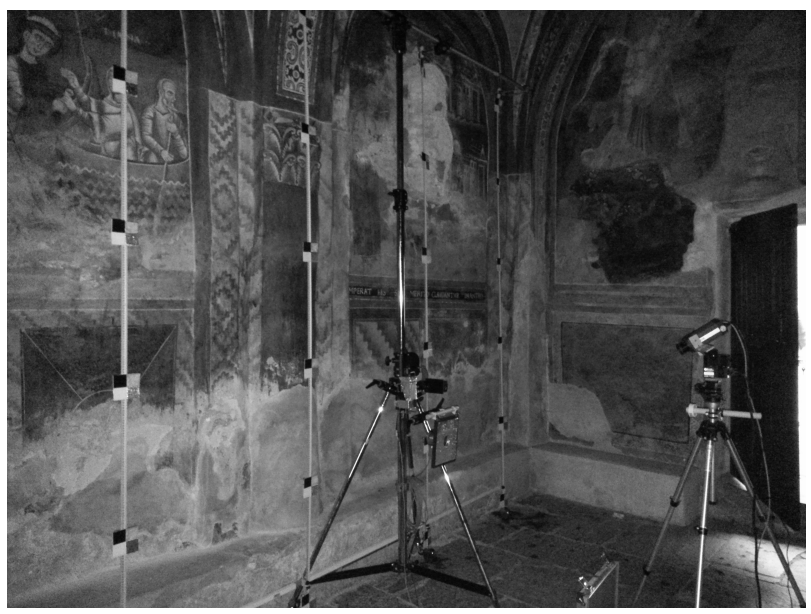

Fig. 3. This picture shows the South wall decoration damaged in the lower part. On the right the robotized system looks at the special reference targets placed near the wall. 
The correct temperature of every pixel is automatically calculated considering the ambient radiation and adjusting the emissivity value according to the angle of view when the surfaces are not Lambertian. The system can be remotely programmed (e.g., by computers, tablets, and smartphones), and it works autonomously in continuous run mode. This feature has been exploited to monitor three inside walls of St. Eldrad's chapel (South, West and North) every 15 minutes for more than one week. This sampling rate is considered more than adequate according to the sampling theorem, being the acquisition frequency much greater than the Nyquist frequency for sampling phenomena that can be described as a continuous signal with a period equal to 24 hours. The infrared system was placed in the centre of the building at about $2.5 \mathrm{~m}$ from the South wall. In small buildings like St. Eldrad's chapel this represents a common problem because at this short distance the typical lens available for infrared cameras, that are generally characterized by a quite limited field of view, make difficult to acquire the whole wall surface. The robotized system allows to move the camera and to scan the surface of interest at a proper pixel resolution. In this application the surface is essentially Lambertian and the average emissivity is around 0.90 (evaluation done according to Ref. 25 ). Thermal data of the inside surfaces are integrated with local microclimatic information provided by the aIRview system itself and two data loggers monitoring the thermohygrometric conditions inside the room. External data such as temperature, relative humidity and solar radiation are supplied by Regional Agency for the Protection of the Environment of Piemonte (ARPA), measured with a weather station and other air temperature and relative humidity probes.

\section{Results}

After processing the data produced by aIRview as FFT amplitude and phase of the South wall, it is possible to clearly distinguish three different horizontal areas, positioned at different heights (A, M and B) as seen in Fig. 4. In particular, the areas labelled A and B show common characteristics, much different from the central M.

The limits of horizontal separation between A and M, and between $\mathrm{M}$ and $\mathrm{B}$ correspond to the most damaged areas of the painted surface, characterized by a strong presence of efflorescence. This occurrence indicates that such areas could be evaporative fronts and this is compatible with the presence of capillary rise phenomena.

According to the mathematical model and the FEM simulation results, the Amplitude and Phase images allow us to advance the hypothesis that $\mathrm{M}$ area corresponds to a wetter wall condition compared to the adjacent $\mathrm{A}$ and $\mathrm{B}$. The average values of the areas are compared in Table 3 . The higher area on the right side of the wall is excluded from the analysis due to its reduced thickness with respect to the rest of the wall.

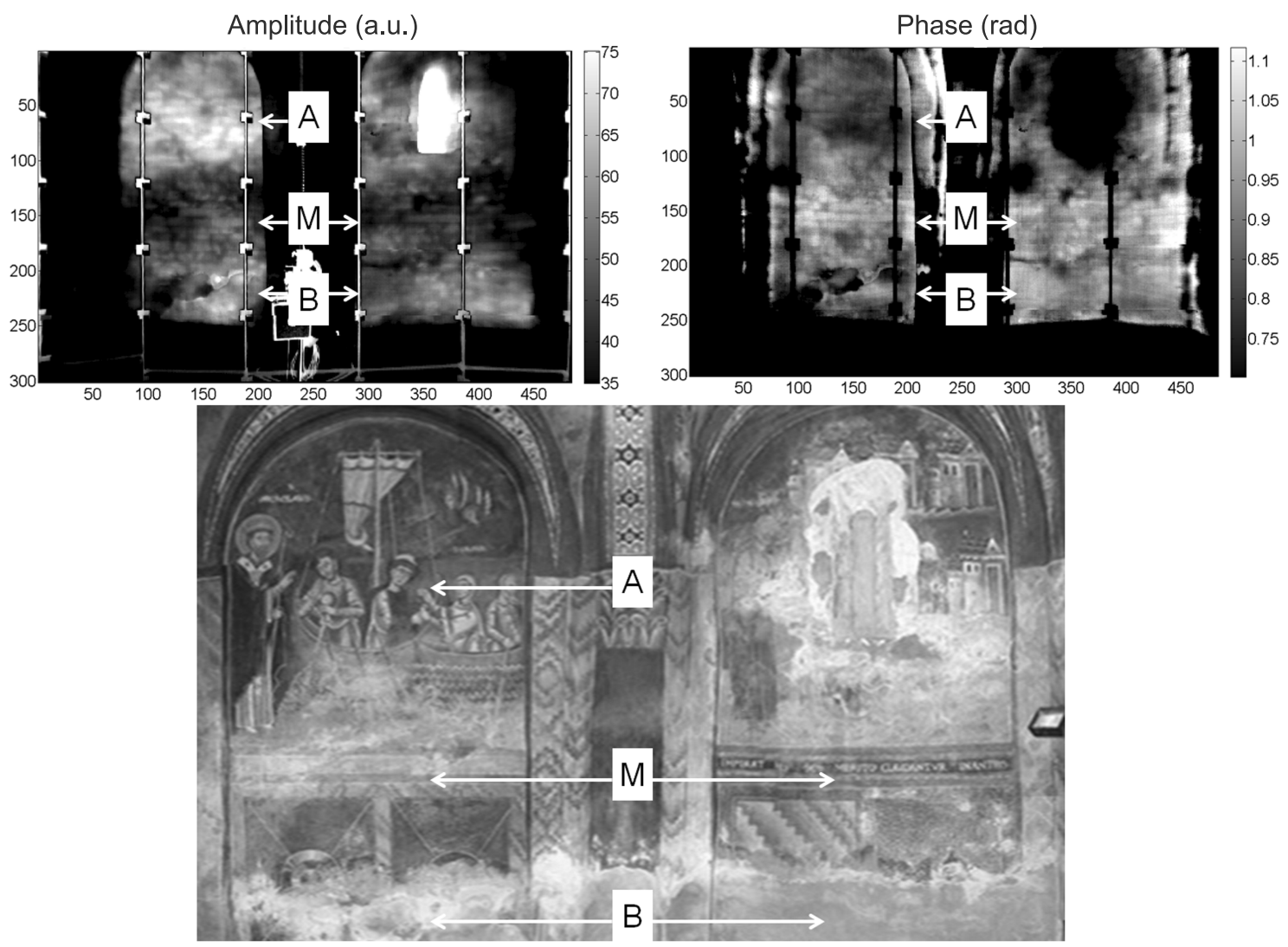

Fig. 4. Amplitude (left) and Phase (right) images. The results show three different horizontal areas, positioned at different heights (A, M and B) with different behaviour, not related to the surface coating visible in the photograph (bottom). 
Table 3. Amplitude and Phase values show that area $M$ is attenuated and phase shifted with respect to the $\mathrm{A}$ and $\mathrm{B}$ areas. This can be attributed to higher moisture content, as described in the previous sections.

\begin{tabular}{ccc}
\hline Area & Amplitude (a.u.) & Phase (rad) \\
\hline A & 54.4 & 0.822 \\
M & 44.4 & 0.963 \\
B & 62.2 & 0.843 \\
\hline
\end{tabular}

\section{Conclusions}

The presence of water in the walls is one of the major risks for the conservation of mural artworks. Related phenomena such as efflorescence and subflorescence of salts take place in the presence of repeated cycles of wetting and drying inside the masonry which involve processes of solvation and crystallization. Two different heights of decay can be identified, both on North and South walls; but more evident on the latter. This is probably due to the cycle of wet and dry that is more emphasized in the South wall that is subjected to the direct solar radiation.

This work represents an effort towards the combination of some data acquisition and signal processing methods typical of the industrial NDE applications with an advanced infrared acquisition system. This is intended to enhance the standard infrared thermography survey of historical building and artworks. The early outcomes of this long term project show how timed acquisitions of wide areas can be useful to study certain phenomena that are otherwise impossible to detect with static or time limited acquisitions. The chosen case study has been particularly challenging due to its peculiar context that required reliable hardware. By analysing the South (damaged) wall as a FFT amplitude and phase images, it has been possible to advance hypotheses that explain the decay mechanism related to moisture presence. A complementary campaign to be conducted in the summer season, during thermal stable weather conditions and sunny days, should provide even more reliable results that will help to understand the phenomena that cause the damage. Furthermore, seasonal measurement campaigns could give information about possible changes in the pattern of the moistened areas and regarding the repeatability of the presented experimental procedure. Future work should include more sophisticated comparisons with software simulation that will take into account real recorded data as an input and local microclimatic information (i.e., air moisture contribution from tourists and air flows from leaking windows). An advance of the aIRview system will be the integration of a visible camera in order to enhance the visualization and interpretation of outputs.

\section{References}

1. C. Rodriguez-Navarro and E. Doehne, "Salt weathering: Influence of evaporation rate, super-saturation and crystallization pattern", Earth Surf. Proc. Land. 24, 191-209 (1999).
2. D. Camuffo, Microclimate for Cultural Heritage: Conservation, Restoration, and Maintenance of Indoor and Outdoor Monuments, Elsevier, New York, 2013.

3. H.E. Silva and F.M. A. Henriques, "Microclimatic analysis of historic buildings: A new methodology for temperate climates", Build. Environ. 82, 381-387 (2014).

4. UNI 11085:2003 Beni culturali-Materiali lapidei naturali ed artificiali-Determinazione del contenuto d'acqua: Metodo ponderale. (In Italian).

5. EN 15758:2010 Conservation of cultural property. Procedures and instruments for measuring temperatures of the air and the surfaces of objects.

6. X. Maldague, Theory and Practice of Infrared Technology for Nondestructive Testing, Wiley, Hoboken, 2001.

7. H. Budzier and G. Garlach, Thermal Infrared Sensors, Theory, Optimization and Practice, John Wiley \& Sons, Hoboken, 2011.

8. S. Bagavathiappan, B.B. Lahiri, T. Saravanan, John Philip, and T. Jayakumar, "Infrared thermography for condition monitoring - A review", Infrared Phys. Techn. 60, 35-55 (2013).

9. C. Meola, Infrared Thermography Recent Advances and Future Trends, Bentham eBooks, 2012.

10. E. Grinzato and E. Rosina, "Infrared and thermal testing for conservation of historic buildings", in Nondestructive Testing Handbook, vol. 3, Infrared and Thermal Testing, X. Maldague ed., ASNT, 2001.

11. E. Grinzato, N. Ludwig, G. Cadelano, M. Bertucci, M. Gargano, and P. Bison, "Infrared thermography for moisture detection: a laboratory study and in-situ test", Mater. Eval. 69, 97-104 (2011).

12. D. Gavrilov, R.G. Maev, and D.P. Almond, "A review of imaging methods in analysis of works of art: Thermographic imaging method in art analysis", Can. J. Phys. 92, 341-364 (2014).

13. G.M. Carlomagno and C. Meola, "Comparison between thermographic techniques for frescoes", NDT \& E Intern. 35, 559-565 (2002).

14. P. Bison, A. Bortolin, G. Cadelano, G. Ferrarini, F. López, and X. Maldague, "Comparison of image processing techniques for the on-site evaluation of damaged frescoes", Proc. SPIE 9105, 91050E (2014).

15. S. Lagüela, J. Martínez, J. Armesto, and P. Arias, "Energy efficiency studies through 3D laser scanning and thermographic technologies", Energ. Buildings, 43, 1216-1221 (2011).

16. M.V. Wyawahare, P.M. Patil, and H.K. Abhyankar, "Image registration techniques: an overview", Intern. J. Signal Process., Image Process. Pattern Recogn. 2, 11-28 (2009).

17. G. Busse, D. Wu, and W. Karpen, "Thermal wave imaging with phase sensitive modulated thermography", J. Appl. Phys. 71, 3962-3965 (1992).

18. S. Torquato, Random Heterogeneous Materials - Microstructure and Macroscopic Properties, Springer-Verlag, New York, 2002.

19. F. Ochs, W. Heidemann, and H. Müller-Steinhagen, "Effective thermal conductivity of moistened insulation materials as a function of temperature", Intern. J. Heat Mass Transfer 51, 539-552 (2008).

20. M.T. Klein, C. Ibarra-Castanedo, X.P. Maldague, and A. Bendada, "A straightforward graphical user interface for ba- 
sic and advanced signal processing of thermographic infrared sequences", Proc. SPIE 6939, 693914-1-9 (2008).

21. A. Bortolin, G. Cadelano, G. Ferrarini, P. Bison, F. Peron, and $X$. Maldague, "High resolution survey of buildings by lock-in IR thermography", Proc. SPIE 8705 (2013).

22. Automation Creations, Inc., MatWeb, Your Source for Materials Information, http://www.matweb.com/, (2009).

23. Comsol Multiphysics, version 4.2, 2011. http://www. comsol.com.
24. P. Bison, A. Bortolin, G. Cadelano, G. Ferrarini, K. Furlan, and E.Grinzato, "Geometrical correction and photogrammetric approach in thermographic inspection of buildings", QIRT, Quantitative InfraRed Thermography, Naples, 11-14 ${ }^{\text {th }}$ June 2012.

25. R.P. Madding, "Emissivity measurement and temperature correction accuracy considerations", Proc. SPIE 3700, 393 (1999). 